\title{
Optimización del proceso de elaboración del bollo de yuca en el municipio de San Juan Nepomuceno, Bolívar-Colombia
}

\author{
Clemente Granados Conde ${ }^{1}$, Estefanía Sarabia Caffroni ${ }^{2}$, Edgar Granados \\ Llamas $^{3}$
}

1 Programa de Ingeniería de Alimentos. Facultad de Ingeniería. Grupo de Investigación Ingeniería, innovación, Calidad Alimentaria y Salud (INCAS), Universidad de Cartagena. Bolívar. Colombia. 2 Ingeniera de Alimentos Universidad de Cartagena.3 Ingeniero Industrial, Universidad de Pamplona. Grupo de investigación Incas, Bolívar, Colombia. Email: clementecondeg@gmail.com.

\section{Resumen}

El objetivo de ésta investigación fue optimizar el proceso de elaboración del bollo de yuca, el cual es un producto autóctono de la costa caribe colombiana y se procesa en el municipio de San Juan de Nepomuceno, departamento de Bolívar, donde se evaluó el proceso actual y el propuesto con el fin de verificar su calidad, aceptación, estudio de tiempos y movimientos. Los resultados obtenidos establecieron que ambos bollos presentaron un nivel de calidad microbiológica de acuerdo a lo establecido en la NTC 4519; el proceso propuesto presentó un menor recuento microbiano debido a que se estableció control higiénico sanitario. En lo referente a la evaluación sensorial el proceso propuesto favoreció la aceptabilidad del bollo de yuca, con altos valores de olor, sabor y color respectivamente, la textura del bollo de yuca artesanal fue el de mayor aceptación; en el aspecto fisicoquímico el bollo de yuca propuesto presentó mejores características como un incremento en proteínas y fibras. En el estudio de tiempos y movimientos, estos se disminuyeron un $24.4 \%$ en tiempo y un $33 \%$ en movimientos, los cuales optimizaran el proceso y disminuirán los costos.

Palabras Clave: Calidad alimentaria, estandarización, calidad, productos autóctonos,material prima e insumos.

\section{Optimization of the process of elaboration of the bollo de yuca in the municipality of San Juan Nepomuceno, Bolívar-Colombia.}

\begin{abstract}
The objective of this research was to streamline the process of elaboration of the bun of cassava, which is an indigenous product of the Colombian Caribbean coast and is processed in the municipality of San Juan Nepomuceno, Bolívar department, where the current process and the proposed was evaluated in order to check their quality, acceptance, study times and movements. The results established that both rolls showed a level of microbiological quality according to the NTC 4519; the proposed process presented a lower microbial count since established sanitary hygienic control. In relation to sensory evaluation the proposed process favored the acceptability of the bun of cassava, with high values of odor, taste and color respectively, Yucca artisan bun texture was greater acceptance; on the physico-
\end{abstract}


chemical aspect proposed Yucca bun presented best features as an increase in protein and fiber. In the study of times and movements, these decreased a $24.4 \%$ in time and $33 \%$ in movements, which will optimize the process and decrease costs.

Keywords: food quality, standardization, quality, local products, raw material and inputs

*Para citar este artículo: Granados Conde C, Sarabia Caffroni E, Granados Llamas E.Optimización del proceso de elaboración del bollo de yuca en el municipio de San Juan Nepomuceno, Bolivar-Colombia. Revista Bistua. 2015;13(2):112-120

+ Autor para el envió de correspondencia y la solicitud de las separatas: Clemente Granados Conde. Programa de Ingeniería de Alimentos. Facultad de Ingeniería. Grupo de Investigación Ingeniería, Innovación, Calidad Alimentaria y Salud (INCAS), Universidad de Cartagena.Bolívar. Colombia.. email: clementecondeg@gmail.com. 


\section{Introducción}

La industria agroalimentaria tradicional se caracteriza por explorar la diversidad, riqueza y costumbres pertenecientes a un territorio, para darle valor agregado a los productos autóctonos e impulsar estos productos alimenticios. Actualmente el fomento de los productos tradicionales presenta un elevado interés, debido a que se basa en el fomento de la comercialización de los productos autóctonos o típicos por medio de la interacción de los parámetros de diferenciación y territorio, su variedad y calidad ${ }^{1}$. Considerando la importancia relevante de éstos productos y el actual interés por la recuperación y fomento, los cuales incrementan el desarrollo socioeconómico del país con productos típicos innovadores y de óptima calidad; los productos elaborados en una determinada zona productora pueden ser competitivos y satisfacer mercados nacionales y globalizados, dando importancia a la calidad, diferenciación y territorio, los cuales están correlacionados ${ }^{1}$ y aportan al consumidor: satisfacción, servicio, seguridad y salud; que se adapten a sus gustos ${ }^{2}$ y requerimientos, o que le evoque un determinado lugar o época ${ }^{3}$. El objetivo de ésta investigación fue optimizar el proceso de la elaboración del bollo de yuca, el cual es un producto autóctono de la costa caribe y que se elabora en el municipio de San Juan de Nepomuceno, departamento de Bolívar, donde se evaluó la calidad del producto actual y propuesto, estudio de tiempos y movimientos.

\section{MATERIALES Y MÉTODOS}

La yuca (Manihot esculenta) pertenece a la familia Euphorbiaceae utilizada en la elaboración del bollo de yuca fue adquirida en el municipio de San Juan de Nepomuceno el coco, azúcar, anís, panela y otros ingredientes fueron obtenidos en el mercado local del municipio y supermercados de Cartagena, Colombia, los cuales fueron utilizados para la elaboración del producto artesanal en el municipio de San Juan de Nepomuceno (Bolívar), y el producto estandarizado en las instalaciones de las plantas Piloto del programa de Ingeniería de Alimentos de la universidad de Cartagena, sede Campus Piedra de Bolívar, Cartagena, Bolívar; donde se establecieron los sistemas de control de procesos ${ }^{4}$, control de calidad, estudios de tiempos y movimientos ${ }^{5}$, perfil sanitario y procesamiento ${ }^{6}$.

\section{Método}

Recepción e inspección de materia prima: la materia prima utilizada en el procesamiento del bollo, se obtuvo de fincas productoras de yuca ubicadas a las afueras del municipio de San Juan de Nepomuceno y que son transportadas diariamente para su comercialización en los Montes de María. La inspección de la yuca se llevó a cabo teniendo en cuenta la NTC 1255 dando cumplimiento a los parámetros establecidos para yuca destinada al consumo humano.

La formulación utilizada se describe en la tabla 1. Las hojas de plátano destinadas para el empaque de los bollos se compraron en tiendas del municipio.

Tabla 1 Formulación elaboración bollo de yuca

\section{Materiales}


FORMULACION ELABORACION BOLLO DE YUCA

\begin{tabular}{|c|c|}
\hline INGREDIENTES & $\begin{array}{c}\text { PORCENTAJE } \\
\%\end{array}$ \\
\hline Yuca & $62.0 \%$ \\
\hline Coco & $15.5 \%$ \\
\hline Azúcar & $14.4 \%$ \\
\hline Sal & $2.33 \%$ \\
\hline Anís & $0.62 \%$ \\
\hline Panela & $7.8 \%$ \\
\hline Total & $\mathbf{1 0 0} \%$ \\
\hline
\end{tabular}

Fuente: Los Autores, 2015

Pesaje: la yuca, el azúcar, sal, anís y panela, fueron pesados en una balanza electrónica modelo Adventurer pro AV 8101 ubicada en las instalaciones de las Plantas Piloto de la Universidad de Cartagena.

Estandarización y mezcla: ésta operación se realizó utilizando un mezclador marca Inducol Amp obteniendo una mezcla homogénea, a partir de la masa de yuca, coco, azúcar, sal, anís y panela, como muestra la figura 1.

Empaque: esta operación se ejecutó utilizando las hojas de plátano como empaque, previamente lavadas.

Cocción: La operación de cocción se efectuó con agua hirviendo a $100^{\circ} \mathrm{C}$, por un tiempo 1:30 minutos.

Escurrido y Almacenamiento: LoS bollos se retiraron y se colocan en recipientes con perforaciones que facilitaron su escurrido y enfriamiento. Terminado el proceso se almacenaron para su comercialización.

Diagrama de proceso de flujo: el diagrama de proceso de flujo es una representación gráfica de la secuencia de todas las operaciones, transportes inspecciones, esperas y almacenamiento que ocurren durante un proceso, incluye además la información que se considera deseable para el análisis, por ejemplo el tiempo necesario y la distancia recorrida, así como las entradas de materia prima y materiales que se utilizan en el proceso de fabricación de los productos. Los diagramas se realizaron de acuerdo al proceso actual y el propuesto (figura 2 y 3 ).

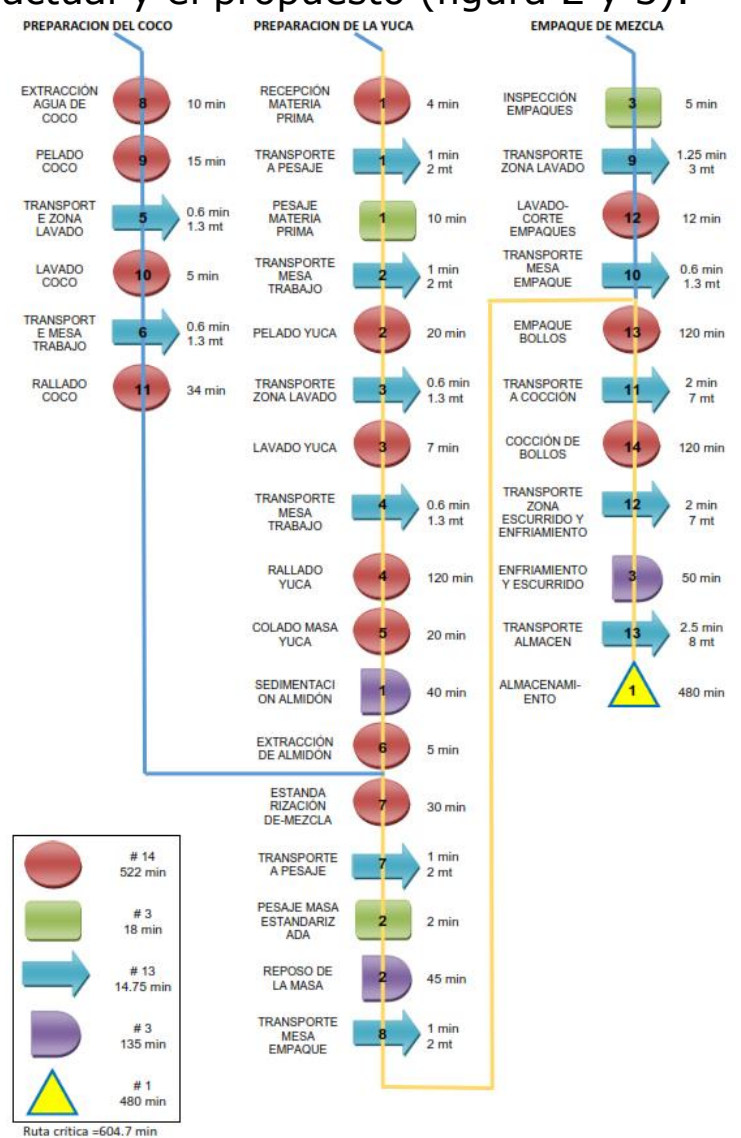

Figura 1. Diagrama de proceso de flujo actual.

Para este lote se procesaron $40 \mathrm{~kg}$ de yuca para obtener 220 bollos. La información necesaria para la elaboración del diagrama se obtuvo a partir de la observación y de la medición directa. 


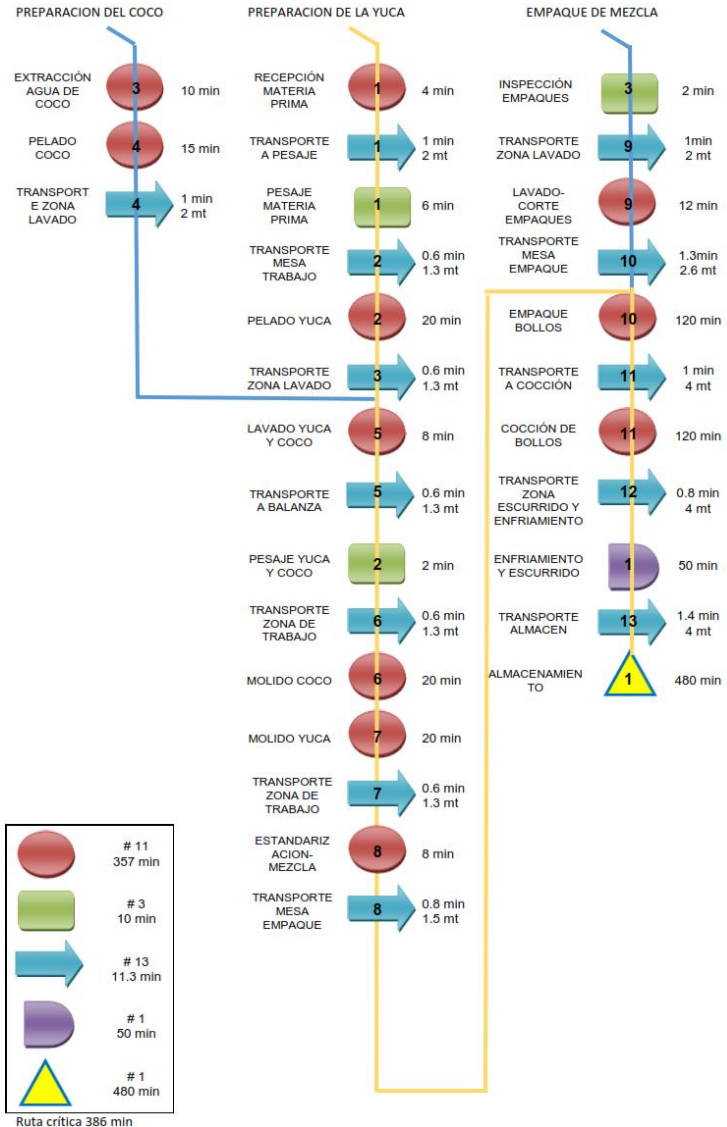

Figura 2. Diagrama de proceso de flujo propuesto.

Distribución de la planta: para esta distribución se tuvo en cuenta el área de producción, pasillo principal y área de operación detallando las zonas de actividades, el modelo matemático utilizado fue "distribución sistemática de múltiples elementos" Ilevando acabo los 6 pasos para hacer una distribución a escala como se observa en la figura 3.

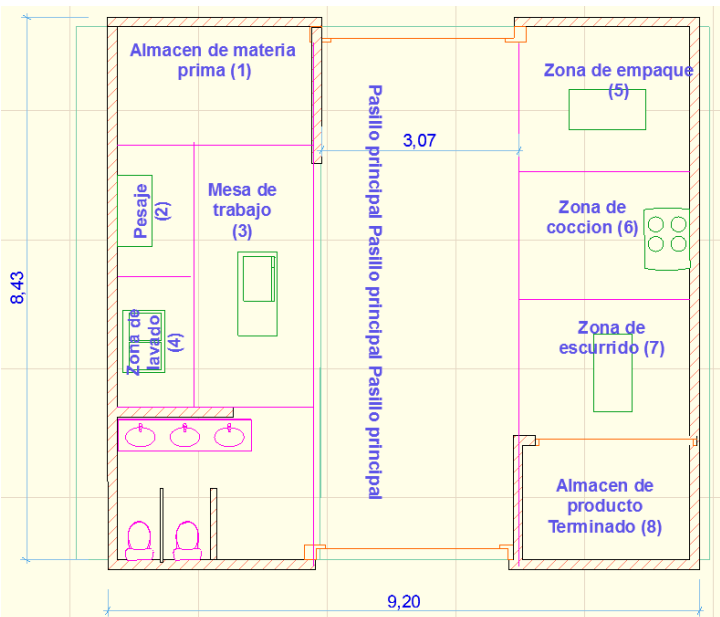

Figura 3. Distribución a escala 1:150

Análisis

Fisicoquímicos,

Microbiológicos y Sensoriales del producto.Análisis bromatológicos: los análisis realizados fueron $\mathrm{pH}$ (Valoración potenciométrica A.O.A.C. $981.12,1990)$, acidez Método titrimétrico A.O.A.C. $942.05,1990)$ y grados Brix Valoración con refractómetro A.O.A.C. 942.33 (1990), pérdida de humedad con relación al tiempo.

Análisis microbiológicos: Las pruebas Microbiológicas realizadas fueron: mesófilos totales ( $\mathrm{ufc} / \mathrm{g} / \mathrm{ml}$ ) coliformes totales ( $\mathrm{ufc} / \mathrm{g} / \mathrm{ml}$ ), coliformes fecales (ufc/g/ml), de acuerdo a la NTC $4519^{7,8}$.

Evaluaciones sensoriales: Se realizaron degustaciones a 40 panelistas consumidores adultos se utilizó una escala hedónica en donde se evaluaron los parámetros como: color, sabor, textura y aceptación general $\left.\right|^{9,10}$. 


\section{RESULTADOS Y DISCUSIÓN Análisis físico químico, bromatológico y microbiológico bollo de yuca.}

Los resultados obtenidos se muestran en la tabla 2. se observa en los ${ }^{\circ}$ Brix que el contenido de sólidos solubles en el bollo de yuca artesanal (actual) es menor que el bollo de yuca propuesto, esto probablemente se debió a la severidad del tratamiento térmico aplicado a cada una de las muestras, ya que en el bollo de yuca propuesto se utilizaron temperaturas controladas a diferencia en el bollo de yuca artesanal en donde se utilizaron temperaturas de ebullición y no controladas, lo cual puede llegar a afectar la cantidad de sólidos solubles presentes en cada una de las muestras.

Tabla 2 Análisis Fisicoquímicos

\begin{tabular}{|l|c|c|l|}
\hline PARAMETROS & \multicolumn{2}{|c|}{ Muestra } & Normas \\
\hline & $\begin{array}{c}\text { Bollo de } \\
\text { yuca actual }\end{array}$ & $\begin{array}{c}\text { Bollo de yuca } \\
\text { propuesto }\end{array}$ & \\
\hline Grados Brix,\% & $3,30 \pm 0,02$ & $4,10 \pm 0,01$ & AOAC 983.17 \\
\hline $\begin{array}{l}\text { Acidez,\% como } \\
\text { ácido sulfúrico }\end{array}$ & $0,05 \pm 0,01$ & $0,05 \pm 0,01$ & TITULACION \\
\hline pH, unidades & $6,81 \pm 0,02$ & $6,79 \pm 0,02$ & $\begin{array}{l}\text { POTENCIOME- } \\
\text { TRICO }\end{array}$ \\
\hline
\end{tabular}

Fuente: Los Autores, 2015

Con relación al pH de los bollos de yuca (artesanal y propuesto) no hubo diferencias significativas $(6,81 \pm 0,02$ y $6,79 \pm 0,02$, ) debido a las condiciones muy similares de procesamiento $y$ sistemas de control.

Los resultados obtenidos en los análisis fisicoquímicos, el bollo de yuca propuesto presentó unos valores más altos en: carbohidratos, proteínas, grasa, fibra total y minerales como se observa en las tablas 2 y 3 , con respecto al bollo de yuca artesanal.

\section{Tabla 3 Análisis Bromatológicos}

\begin{tabular}{|l|l|l|l|}
\hline Parámetros & \multicolumn{2}{|c|}{ Muestra } & Normas \\
\hline & $\begin{array}{l}\text { Bollo de } \\
\text { yuca actual }\end{array}$ & $\begin{array}{l}\text { Bollo de yuca } \\
\text { propuesto }\end{array}$ & \\
\hline $\begin{array}{l}\text { Carbohidra- } \\
\text { tos }\end{array}$ & $\begin{array}{l}46,29 \quad \pm \\
0,28\end{array}$ & $49,06 \pm 0,32$ & $\begin{array}{l}\text { Diferencia según } \\
\text { FAO y OMS }\end{array}$ \\
\hline Proteínas & $1,27 \pm 0,02$ & $1,76 \pm 0,04$ & $\begin{array}{l}\text { AOAC } 984.13 \\
\text { KJELDAHL }\end{array}$ \\
\hline Grasas & $0,20 \pm 0,01$ & $0,22 \pm 0,01$ & AOAC 920.39C \\
\hline Fibra total & $0,33 \pm 0,01$ & $0,65 \pm 0,02$ & AOAC 962.09 \\
\hline Minerales & $0,66 \pm 0,05$ & $1,35 \pm 0,07$ & AOAC 923.03 \\
\hline Humedad & $\begin{array}{l}48,26 \\
0,27\end{array}$ & $49,55 \pm 0,34$ & AOAC 964.22 \\
\hline
\end{tabular}

Fuente: Los Autores, 2015

Estudios realizados ${ }^{12}$ confirman que el bollo de yuca provee grandes cantidades de energía al cuerpo en forma de carbohidratos ${ }^{13}$. Se puede afirmar que el valor de minerales se atribuye a la presencia de calcio y el fósforo contenidos en la yuca ${ }^{14}$. El contenido de humedad en ambas muestras de bollos es significativamente bajo aunque estas condiciones pueden favorecer la proliferación de microorganismos patógenos ${ }^{12}$. Los resultados obtenidos en la prueba de análisis microbiológico resalta la presencia de microorganismos en ambos bollos, teniendo en cuenta que el bollo de yuca propuesto es apto para el consumo humano por encima del bollo de yuca elaborado artesanalmente.

De acuerdo a los diferentes criterios contemplados en el decreto 3075, la empresa "Bollería San Juan" obtiene $62 \%$ en perfil sanitario, donde sus principales deficiencias son las instalaciones físicas, control de calidad procesamiento y almacenamiento, los cuales inciden en la calidad del producto final debido a: las condiciones higiénico sanitarias de ésta planta procesadora ${ }^{16}$, personal manipulador, 
empaque $y$ condiciones de almacenamiento ${ }^{17}$.

Adicionalmente, los bollos de yuca presentaron recuentos de aerobios mesófilos que están dentro de los parámetros normales; siendo los mayores recuentos en el bollo de yuca artesanal, microorganismos que son capaces de desarrollarse a $30^{\circ} \mathrm{C}$ en las condiciones establecidas; aun cuando estos microorganismos no son conocidos como patógenos y alteran las propiedades organolépticas del alimento, lo cual podría ser por deficiencia del procedimiento de elaboración en el proceso, la condición de higiene de los equipo y utensilios y la relación tiempo / temperatura de almacenamiento y distribución del producto $^{14}$. Analizando la presencia de coliformes totales y fecales en el bollo de yuca artesanal, éste presenta un recuento alto de coniformes totales y valores por debajo de lo establecido dentro de coliformes fecales. Por su parte, el bollo de yuca propuesto presenta valores más bajos, lo cual indica que hubo contaminación del bollo artesanal en la etapa del proceso, ya sea por contacto con agua $y$ alimentos contaminados $\mathrm{y} / \mathrm{o}$ falta de higiene ${ }^{4}$.

La presencia de microorganismos en el bollo podría atribuirse al tipo de empaque utilizado (hojas de plátano) en los procesos envoltura, cocción y empaque final en las muestras, ya que estas no protegen totalmente el bollo y no sirven como una barrera contra microorganismos, lo que conlleva a que sea un medio para la proliferación de los mismos y de esta manera se evidencia la rápida alteración microbiológica de los bollos de yuca durante el almacenamiento.

\section{Tabla 4 Análisis Microbiológicos}

\begin{tabular}{|c|c|c|c|c|}
\hline $\begin{array}{c}\text { TIPO DE } \\
\text { MUESTRA }\end{array}$ & $\begin{array}{c}\text { MESOFILOS } \\
\text { AEROBIOS } \\
\text { Ufc/g }\end{array}$ & $\begin{array}{c}\text { COLIFORMES } \\
\text { TOTALES } \\
\text { Ufflg }\end{array}$ & $\begin{array}{c}\text { COLIFORMES } \\
\text { FECALES } \\
\text { Ufc/g }\end{array}$ & $\begin{array}{c}\text { MOHOS Y } \\
\text { LEVADU } \\
\text { RAS }\end{array}$ \\
\hline V. referencia & 10.000 ufc/g & $<10 \mathrm{ufc} / \mathrm{g}$ & $<10 \mathrm{ufclg}$ & $100 \mathrm{ufclg}$ \\
\hline $\begin{array}{c}\text { Bollo de } \\
\text { yuca } \\
\text { artesanal }\end{array}$ & $7300 \mathrm{ufc} / \mathrm{g}$ & $>3000 \mathrm{ufc} / \mathrm{g}$ & $<10 \mathrm{ufc} / \mathrm{g}$ & $25 \mathrm{ufc} / \mathrm{g}$ \\
\hline $\begin{array}{l}\text { Bollo de } \\
\text { yuca } \\
\text { propuesto }\end{array}$ & $200 \mathrm{ufc} / \mathrm{g}$ & $<10 \mathrm{ufc} / \mathrm{g}$ & $<10 \mathrm{ufc} / \mathrm{g}$ & $\begin{array}{r}8 u \\
\mathrm{fc} / \mathrm{g}\end{array}$ \\
\hline
\end{tabular}

Fuente: Los Autores, 2015

En la actualidad $^{16}$ la inocuidad de los alimentos establece que los microorganismos se cuantifican para determinar la calidad sanitaria de alimentos tales como el bollo de yuca.

Estudio de tiempos y movimientos En la tabla 5, se observa que en lo referente al estudio de tiempos y movimientos en el proceso actual y propuesto, estos disminuyeron un 26 $\%$ con respecto al tiempo y un 34\% con relación al movimiento en todas las operaciones.

Tabla 5 Comparativo Métodos Elaboración bollo de yuca

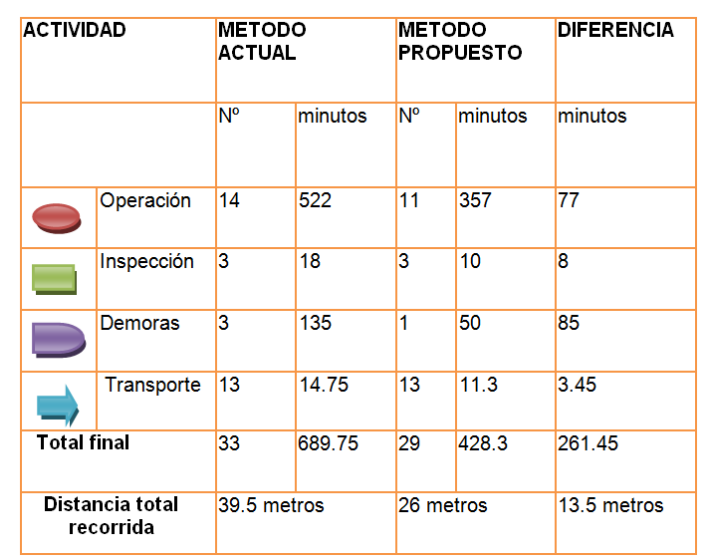

Fuente: Los Autores, 2015

Los factores que pudieron incidir a la disminución de estos tiempos se puede atribuir a un mayor tiempo de cocción efectuada en el bollo de yuca artesanal, el cual lo realizan en fogón 
de leña lo que acarrea un mayor tiempo en esta etapa, sin embargo se consideraron un mayor número de inspecciones en el proceso de bollo de yuca propuesto; las demoras pasaron de 3 en el bollo de yuca artesanal a 1 en el bollo de yuca propuesto, por otra parte, el reposo de la masa final antes del empaque de los bollo de yuca en el proceso artesanal, no evidencia ningún factor influyente o característico en el producto final, por lo cual en el bollo de yuca propuesto se descarta esta etapa, teniendo en cuenta que en el bollo de yuca artesanal esta fase se lleva a cabo por satisfacer aspectos culturales. El número de transportes aumentaron en el bollo de yuca propuesto aunque la distancia y los tiempos empleados disminuyeron ${ }^{19}$, lo cual permitió mejorar éste proceso ${ }^{20}$.

\section{Evaluación sensorial}

En lo referente al color, olor y sabor del bollo de yuca propuesto presentó una mayor aceptación, en lo que respeta a la textura tuvo más aceptación en el bollo artesanal. Teniendo en cuenta que desde el punto de vista de la calidad de los alimentos, la inocuidad es fundamental, estos deben cumplir atributos de calidad organoléptica que condicionan la aceptabilidad del bollo de yuca de acuerdo a su capacidad de actuar sobre los sentidos.

\section{Conclusiones}

La optimización de éste proceso permitió obtener un mejor producto. Los análisis microbiológicos del proceso artesanal propuesto de bollo de yuca presentaron un menor recuento microbiano en lo referente a: recuento de coliformes totales, aerobios mesófilos, mohos y levaduras. Los análisis fisicoquímicos y bromatológicos obtenidos en los bollos 119 de yuca, artesanal y propuesto fueron muy similares debido a que su formulación y procesamiento fueron muy semejantes.

La evaluación sensorial aplicada favorece la aceptabilidad del bollo de yuca propuesto, con altos valores de olor, sabor y color respectivamente, la textura del bollo de yuca artesanal fue la de mayor aceptación.

Con relación al estudio de tiempos y movimientos en la producción del bollo de yuca en el método propuesto se disminuyeron en un $26 \%$ en tiempo y $34 \%$ en movimientos respectivamente, los cuales mejoran la línea de producción del bollo de yuca en lo referente a su calidad y bajo costo.

\section{Referencias bibliográficas}

1. Bello Gutiérrez J. Calidad de vida, Alimentos y Salud Humana: Fundamentos científicos. Ediciones Díaz de Santos. España: Health \& Fitness; 2012.

2 Caldentey P, Gómez A. Slowood: Agricultura y gastronomía. Agricultura: Rev. Agropecuaria. LXXVI, 2007; 895: $308-310$.

3 Maypa. Libro blanco en Agricultura y Desarrollo Rural. Madrid, Ministerio de Agricultura, Pesca y Alimentación. [Consulta: 20-09-2009]. Disponible en http://www.libroblancoagricultura.com/

4 Torrenegra $\mathrm{M}$, et al. Characterization of the preparation process of clean corn bollo in Villanueva Municipality (BolivarColombia). Biotecnología en el Sector Agropecuario y Agroindustrial. 2013; 11 (2):148-155.

5 Niebel $B$, Freivalds A. Ingeniería Industrial, Métodos, Tiempos y 
Movimientos. Décima edición. México, D.F: Editorial: Alfa omega; 2001.

6 Torres $\mathrm{P}$, et al. Una mirada a la agroindustria de extracción de almidón de yuca, desde la estandarización de procesos. Revista EIA. 2010; 14:3-5.

7 Norma Técnica Colombiana. Yuca fresca NTC.4519. Instituto Colombiano de Normas Técnicas .Santafé de Bogotá, Colombia; (1979).

8 Frazier W, Westhoff D. Microbiología de los Alimentos. Cuarta edición Zaragoza, España: Editorial Acribia; 1993.

9 Anzaldua Morales A. La Evaluación Sensorial de los Alimentos en la Teoría y Práctica. Zaragoza España: Editorial Acribia; 2005.

10 Carpenter $\mathrm{R}$, Lyon $\mathrm{P}$, Hasdell $\mathrm{D} \mathrm{H}$. Análisis sensorial en el desarrollo y control de la calidad de alimentos. Zaragoza España: Editorial Acribia; 2009.

11 Meyers, Fred E. Estudio de tiempos y movimientos para la manufactura ágil. Segunda Edición. México: Editorial Pearson Educación; 2000.

12 Rodríguez $\mathrm{O}$ et al. Simulación y optimización de un proceso de transferencia de calor para la inactivación de clostridium botulinum en alimentos colombianos Ibagué. Universidad del Tolima. Facultad de Ciencias de la Universidad del Tolima., 2008. pp. 1-4.

13 Aristizábal J, et al. Guía técnica para producción y análisis de almidón de yuca. Boletín de servicios agrícolas de la FAO. Roma. 2007; 1-3, 19-22.

14 Giraldo A, et al. Digestibilidad aparente de una harina proveniente de hojas de yuca (Manihot esculenta crantz).
Información Tecnológica. 2008; 19 (1) 11-18.

15 Mc allister Tafur G. La inocuidad de alimentos y el comercio internacional. Rev Colom Cienc Pecua. 2009; 22(3):330338.

16 Signorini $M$, et al. Utilización de microorganismos marcadores para la evaluación de las condiciones higiénico sanitarias en la producción primaria de leche. Rev. Cient. 2008; 18 (2) 1-2.

17 Decreto 3075. Ministerio de la protección social. Inicio Normatividad Alimentos Decretos. Resolución 2674 de 2013 que reglamenta parcialmente el capítulo $\mathrm{V}$ (Alimentos) de la ley 9 de 1979, más conocida como Código Sanitario Nacional. 1997.

18 Caballero A. Temas de Higiene de los Alimentos. La Habana, Cuba Editorial Ciencias Médicas; 2008.

19 Torres P. et al. Una mirada a la agroindustria de extracción de almidón de yuca, desde la estandarización de procesos. Revista EIA.2011; 14:23-38.

20 Konz Stephan. Diseño de instalaciones industriales. México: Editorial Limusa; 2004. 